УДК 512.53

\title{
Equationally Extremal Semilattices
}

Artem N. Shevlyakov*

Omsk Branch of Sobolev Institute of Mathematics SB RAS

Pevtsova, 13, Omsk, 644099

Omsk State Technical University

Mira, 11, Omsk, 644050

Russia

Received 02.11.2016, received in revised form 10.12.2016, accepted 20.02.2017

In the current paper we study extremal semilattices with respect to their equational properties. In the class $\mathbf{S}_{n}$ of all semilattices of order $n$ we find semilattices which have maximal (minimal) number of consistent equations. Moreover, we find a semilattice in $\mathbf{S}_{n}$ with maximal sum of numbers of solutions of equations.

Keywords: semilattice, equation, solutions, consistency, universal algebraic geometry. DOI: 10.17516/1997-1397-2017-10-3-298-304.

\section{Introduction and preliminaries}

In the current paper we study equations over finite semilattices. Let $\mathbf{S}_{n}$ denote the class of all semilattices of order $n$. The class $\mathbf{S}_{n}$ contains extremal semilattices relative to their equational properties. For example, $\mathbf{S}_{n}$ contains a semilattice $S$ with a maximal (minimal) number of consistent equations.

Below we study such extremal semilattices from $\mathbf{S}_{n}$. In Theorem 4.1 we find a semilattice with a maximal (minimal) number of consistent equations. The semilattice found is isomorphic to the linearly ordered semilattice (to the fan semilattice, respectively). In Theorem 5.1 we prove that the fan semilattice has a maximal value of

$$
\Sigma(S)=\sum_{\text {eq }}\left|\mathrm{V}_{S}(\mathbf{e q})\right|,
$$

where eq iterates the set of all equations in one variable. Unfortunately, the corresponding semilattice with a minimal sum is not found yet. We can only formulate the following conjecture

Conjecture. Let $\Sigma(S)$ has the minimum value $\Sigma$ in the set $\mathbf{S}_{n}$. Then there exists a semilattice $S_{1} \in \mathbf{S}_{n}$ with a unique co-atom such that $\Sigma\left(S_{1}\right)=\Sigma$.

We discuss this conjecture in Section 6.

\section{Basic notions}

Let $S$ be a semilattice of order $n$, where the meet operation is denoted by $\cdot$ and the minimal element is denoted by 0 . The partial order over a semilattice $S$ is defined by · as follows

$$
x \leqslant y \Leftrightarrow x y=x .
$$

*a_shevl@mail.ru

(c) Siberian Federal University. All rights reserved 
Moreover, we assume that any semilattice below contains the maximal element 1 . This assumption is not strict, since one can adjoint 1 to any semilattice with no maximal element. An element $s \in S$ is called an atom (co-atom) if there is not an element $t \in S$ with $0<t<s$ (respectively, $s<t<1$ ). The set of all atoms of a semilattice $S$ is denoted by $\operatorname{At}(S)$. Let $\operatorname{Anc}(s)$ denote the set of all ancestors of an element $s \in S$, i.e.

$$
t \in \operatorname{Anc}(s) \Leftrightarrow t<s \text { and } \nexists u t<u<s .
$$

Let $S$ be a semilattice and $X=\left\{x_{1}, x_{2}, \ldots, x_{m}\right\}$ a variable set. An equation over $S$ is an ordered pair $\left(t(X) s_{1}, s(X) s_{2}\right)$ or $\left(t(X) s_{1}, s_{2}\right)$, where $t(X), s(X)$ are nonempty products of variables of $X$ and $s_{i} \in S$. Below we shall write equations in traditional form: $t(X) s_{1}=s(X) s_{2}$, $t(X) s_{1}=s_{2}$.

Remark 2.1. Let us give some remarks about the set of equations over $S$ :

1. Since an equation is an ordered pair, the expressions $t(X) s_{1}=s(X) s_{2}, s(X) s_{2}=t(X) s_{1}$ are assumed to be different equations.

2. Formally, the expression $s_{1}=t(X) s_{2}$ is not an equation over $S$, but $t(X) s_{2}=s_{1}$ is.

3. Since $1 \in S$, the following expressions $t(X) s_{1}=s(X), t(X)=s(X) s_{2}, t(X)=s(X)$, $t(X)=s_{2}$ are equations over $S$.

An equation of the form $t(X) s_{1}=s(X) s_{2}\left(t(X) s_{1}=s_{2}\right)$ is called an equation of the first (respectively, second) kind. Let $\operatorname{Eq}_{m}^{i}(S)$ denote the set of all equations of the $i$-th kind in at most $m$ variables over a semilattice $S$.

Let $\operatorname{Eq}_{m}(S)=\operatorname{Eq}_{m}^{1}(S) \cup \mathrm{Eq}_{m}^{2}(S)$ be the set of all equations in at most $m$ variables over a semilattice $S$. For a semilattice $S$ of order $n$ one can directly compute that $\left|\operatorname{Eq}_{m}^{1}(S)\right|=$ $\left(2^{m}-1\right)^{2} n^{2},\left|\mathrm{Eq}_{m}^{2}(S)\right|=\left(2^{m}-1\right) n^{2}$, and finally

$$
\left|\mathrm{Eq}_{m}(S)\right|=\left(2^{m}-1\right)^{2} n^{2}+\left(2^{m}-1\right) n^{2}=2^{m} n^{2}\left(2^{m}-1\right) .
$$

\section{Two series of semilattices}

Let $L_{n}=\left\{a_{0}, a_{1}, a_{2}, \ldots, a_{n-1}\right\}$ be the linearly ordered semilattice of order $n(n \geqslant 2)$. The order over $L_{n}$ is given by

$$
a_{0}<a_{1}<\ldots<a_{n-1} .
$$

Let $F_{n}=\left\{0, a_{1}, a_{2}, \ldots, a_{n-2}, 1\right\}$ be the semilattice consisting of $n(n \geqslant 3)$ elements, and $A_{n}=\left\{a_{i} \mid 1 \leqslant i \leqslant n-2\right\}$ is an anti-chain (Fig. 1).

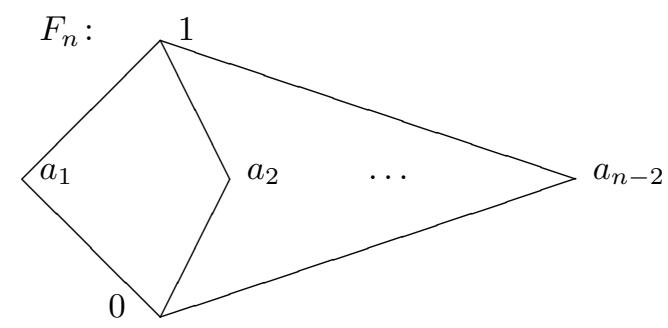

Fig. 1. The semilattice $F_{n}$ 


\section{Inconsistent equations}

In [1] it was described extremal semilattices of order $n$ with minimal (maximal) number of inconsistent equations. Let $\mathrm{Eq}_{m \emptyset}(S)$ be the set of all inconsistent equations over a semilattice $S$ in at most $m$ variables

Theorem 4.1. [1, Theorem 1]. For any semilattice $S$ of order $n$ it holds

$$
\left|\mathrm{Eq}_{m \emptyset}\left(L_{n}\right)\right| \leqslant\left|\mathrm{Eq}_{m \emptyset}(S)\right| \leqslant\left|\mathrm{Eq}_{m \emptyset}\left(F_{n}\right)\right|
$$

and

$$
\left|\mathrm{Eq}_{m \emptyset}\left(L_{n}\right)\right|=\left(2^{m}-1\right) \frac{n(n-1)}{2},\left|\mathrm{Eq}_{m \emptyset}\left(F_{n}\right)\right|=\left(2^{m}-1\right)\left(n^{2}-3 n+3\right) .
$$

Moreover, in [1] it was proved that the empty set is most probable in a random generation of equations. More formally, let $\mathrm{Eq}_{1}(S, Y) \subseteq \mathrm{Eq}_{1}(S)$ be the set of all equations with the solution set $Y \subseteq S$, and we have the following statement.

Theorem 4.2. [1, Theorem 4]. For any semilattice $S$ of order $n \geqslant 6$ and any algebraic subset $Y \subseteq S$ it holds

$$
\left|\mathrm{Eq}_{1}(S, Y)\right| \leqslant\left|\mathrm{Eq}_{1}(S, \emptyset)\right| .
$$

\section{Number of all solutions}

Let us consider an expression

$$
\Sigma(S)=\sum_{\mathbf{e q} \in \mathrm{Eq}_{1}(S)}\left|\mathrm{V}_{S}(\mathbf{e q})\right|
$$

where an equation eq iterates the set of all equations in one variable, and find a semilattice with minimal (maximal) value of $\Sigma(S)$. Firstly, one can directly show that

$$
\Sigma(S)=\sum_{s \in S}|\operatorname{cov}(s)|,
$$

where $\operatorname{cov}(s)=\operatorname{cov}_{1}(s) \cup \operatorname{cov}_{2}(s)$ and $\operatorname{cov}_{i}(s)=\left\{\mathbf{e q} \in \mathrm{Eq}_{1}^{i}(S) \mid s \in \mathrm{V}_{S}(\mathbf{e q})\right\}$.

Lemma 5.1. For any element $s$ of a semilattice $S$ with $|S|=n$ it holds

$$
\operatorname{cov}_{2}(s)=\{x a=b \mid a \in S, b=a s\},\left|\operatorname{cov}_{2}(s)=n\right| .
$$

Proof. An equation $x a=b$ of the second kind is satisfied by $s$ iff $b=a s$. Therefore the right part of the equation is determined by a constant $a$ of the left part. Since $a$ belongs to the $n$-element set $S$, there are exactly $n$ equations in $\operatorname{cov}_{2}(s)$.

Thus, $|\operatorname{cov}(s)|=\left|\operatorname{cov}_{1}(s)\right|+n$ and below we study only the set $\operatorname{cov}_{1}(s)$. Let

$$
\uparrow s=\{t \mid t \geqslant s\}, \perp s=\{t \mid t s=0\} .
$$

Lemma 5.2. For any semilattice $S$ of the order $n$ we have

1)

$$
\begin{gathered}
\operatorname{cov}_{1}(0)=\left\{x a=x a^{\prime} \mid a, a^{\prime} \in S\right\},\left|\operatorname{cov}_{1}(0)\right|=n^{2} \\
\operatorname{cov}_{1}(1)=\{x a=x a \mid a \in S\}\left|\operatorname{cov}_{1}(1)\right|=n ;
\end{gathered}
$$


2) for any $s \in S$

$$
\operatorname{cov}_{1}(s)=\bigcap_{t \in \operatorname{Anc}(s)} \operatorname{cov}_{1}(t)
$$

if $|\operatorname{Anc}(s)|>1$, and

$$
\operatorname{cov}_{1}(s)=\operatorname{cov}_{1}\left(s^{\prime}\right) \backslash\left\{x a=x a^{\prime} \mid a \geqslant s, a^{\prime} \geqslant s^{\prime}, a^{\prime} ¥ s\right\}
$$

if $\operatorname{Anc}(s)=\left\{s^{\prime}\right\}$

3) for any $s \leqslant t$ it holds

$$
\operatorname{cov}_{1}(s) \leqslant \operatorname{cov}_{1}(t)
$$

4) if $a \in \operatorname{At}(S)$ then

$$
\begin{aligned}
& \operatorname{cov}_{1}(a)=\{x s=x t \mid s, t \geqslant a\} \cup\{x s=x t \mid s, t \nsupseteq a\}, \\
& \left|\operatorname{cov}_{1}(a)\right|=|\uparrow a|^{2}+|\perp a|^{2}=|\uparrow a|^{2}+(n-|\uparrow a|)^{2} .
\end{aligned}
$$

Proof.

1. The proof is straightforward.

2. We prove only (5), since the proof of (6) is straightforward. If $x a=x a^{\prime} \in \operatorname{cov}_{1}(s)$ then

$$
s a=s a^{\prime} \Rightarrow t s a=t s a^{\prime} \Rightarrow t a=t a^{\prime} \Rightarrow x a=x a^{\prime} \in \operatorname{cov}_{1}(t)
$$

for any $t \in \operatorname{Anc}(s)$. Thus,

$$
\operatorname{cov}_{1}(s) \subseteq \bigcap_{t \in \operatorname{Anc}(s)} \operatorname{cov}_{1}(t) .
$$

Let us prove the converse inclusion. Suppose $x a=x a^{\prime} \in \bigcap_{t \in \operatorname{Anc}(a)} \operatorname{cov}_{1}(t)$ but $s a \neq s a^{\prime}$. Then there exist $t_{1}, t_{2} \in \operatorname{Anc}(s)$ such that $s a \leqslant t_{1}, s a^{\prime} \leqslant t_{2}$. The equalities $t_{i} a=t_{i} a^{\prime}$ imply

$$
s a=(s a) t_{1}=\left(s t_{1}\right) a=t_{1} a=t_{1} a^{\prime}, s a^{\prime}=\left(s a^{\prime}\right) t_{2}=\left(s t_{2}\right) a^{\prime}=t_{2} a^{\prime}=t_{2} a .
$$

Therefore,

$$
\begin{gathered}
a a^{\prime} s=a^{\prime}(a s)=a^{\prime} t_{1} a^{\prime}=t_{1} a^{\prime}=s a, \\
a a^{\prime} s=a\left(a^{\prime} s\right)=a t_{2} a=t_{2} a=s a^{\prime},
\end{gathered}
$$

and we obtain a contradiction $s a=s a^{\prime}$.

3. Directly follows from the previous statement.

4. The statement immediately follows from formulas $(3,6)$, since $\operatorname{Anc}(s)=\{0\}$ for any atom $s$.

The following lemmas contain results about the sets $\operatorname{cov}_{1}(s)$ in semilattices $L_{n}, F_{n}$.

Lemma 5.3. For each $s \in L_{n}$ we have

$$
\begin{gathered}
\operatorname{cov}_{1}(s)=\left\{x a=x a^{\prime} \mid a, a^{\prime} \geqslant s\right\} \cup\{x a=x a \mid a<s\}, \\
\left|\operatorname{cov}_{1}\left(a_{i}\right)\right|=(n-i)^{2}+i, \\
-301-
\end{gathered}
$$


Lemma 5.4. For each $s \in F_{n}, n \geqslant 4$ we have

$$
\begin{aligned}
\operatorname{cov}_{1}\left(a_{i}\right)=\left\{x a_{j}=x a_{k} \mid j, k \in\{0,1,2, \ldots,\right. & n-2\} \backslash\{i\}\} \\
\cup & \left\{x a_{j}=x a_{k} \mid j, k \in\{i, n-1\}\right\}(i \notin\{1, n\}) . \\
\left|\operatorname{cov}_{1}\left(a_{i}\right)\right| & =(n-2)^{2}+4 .
\end{aligned}
$$

Theorem 5.1. For any semilattice $S$ of order $n \geqslant 4$ we have

$$
\sum_{s \in S}\left|\operatorname{cov}_{1}(s)\right| \leqslant \sum_{s \in F_{n}}\left|\operatorname{cov}_{1}(s)\right| .
$$

Proof. It is clear that for any $s \in S \backslash\{1\}$ we have

$$
|\uparrow s| \geqslant 2 .
$$

Suppose $S$ contains at least two atoms then for each atom $a$ we have

$$
2 \leqslant\left|\perp a_{i}\right| .
$$

and (9) gives

$$
\left|\operatorname{cov}_{1}(a)\right| \leqslant(n-2)^{2}+4
$$

for each $a \in \operatorname{At}(S)$.

By (7), for each $s \in S \backslash\{0,1\}$ it holds

$$
\left|\operatorname{cov}_{1}(s)\right| \leqslant(n-2)^{2}+4
$$

and, using $(3,4,12)$, we have

$$
\sum_{s \in S}\left|\operatorname{cov}_{1}(s)\right|=\left|\operatorname{cov}_{1}(0)\right|+\left|\operatorname{cov}_{1}(1)\right|+\sum_{s \in S \backslash\{0,1\}}\left|\operatorname{cov}_{1}(s)\right| \leqslant n^{2}+n+(n-2)\left((n-2)^{2}+4\right)=\sum_{s \in F_{n}}\left|\operatorname{cov}_{1}(s)\right| .
$$

Suppose now that $S$ has exactly one atom $a$. Then (8) gives

$$
\left|\operatorname{cov}_{1}(a)\right|=(n-1)^{2}+1 .
$$

Let us prove the inequality

$$
\left|\operatorname{cov}_{1}(s)\right| \leqslant(n-2)^{2}+2
$$

for each $s \in S \backslash\{0, a\}$. We have exactly two cases.

1. Indeed, if $a$ has a unique descendant $a^{\prime}$ (i.e. $\operatorname{Anc}\left(a^{\prime}\right)=\{a\}$ ) one can directly compute that

$$
\operatorname{cov}_{1}\left(a^{\prime}\right)=\left\{x b=x c \mid b, c \geqslant a^{\prime}\right\} \cup\{x a=x a, x 0=x 0\},\left|\operatorname{cov}_{1}\left(a^{\prime}\right)\right|=(n-2)^{2}+2,
$$

and, using (7), we obtain (20).

2. Suppose $a$ is the ancestor of $a_{1}, a_{2}, \ldots, a_{k}(k \geqslant 2)$. Then one can compute that

$$
\operatorname{cov}_{1}\left(a_{i}\right)=\left\{x b=x c \mid b, c \geqslant a_{i}\right\} \cup\left\{x b=x c \mid b, c \in \perp_{a} a_{i}\right\} \cup\{x 0=x 0\},
$$

where $\perp_{a} a_{i}=\left\{s \in S \mid s a_{i}=a\right\}$. Thus,

$$
\left|\operatorname{cov}_{1}\left(a_{i}\right)\right|=\left|\uparrow a_{i}\right|^{2}+\left|\perp_{a} a_{i}\right|^{2}+1 .
$$


We have

$$
2 \leqslant\left|\uparrow a_{i}\right| \leqslant n-3,
$$

(since $a_{i}, 1 \in \uparrow a_{i}$ and $0, a, a_{j} \notin \uparrow a_{i}$ for $j \neq i$ ),

$$
2 \leqslant\left|\perp_{a} a_{i}\right| \leqslant n-3,
$$

(since $a, a_{j} \in \perp_{a} a_{i}$ for $j \neq i$, and $0,1, a_{i} \notin \perp_{a} a_{i}$ ). Since $\uparrow a_{i} \cap \perp_{a} a_{i}=\emptyset$ and $0 \notin$ $\left(\uparrow a_{i} \cup \perp_{a} a_{i}\right)$ then

$$
\left|\uparrow a_{i}\right|+\left|\perp_{a} a_{i}\right| \leqslant n-1
$$

By (21-23), one can directly compute that

$$
\left|\uparrow a_{i}\right|^{2}+\left|\perp_{a} a_{i}\right|^{2} \leqslant(n-3)^{2}+2^{2}=n^{2}-6 n+13 .
$$

Therefore,

$$
\left|\operatorname{cov}_{1}\left(a_{i}\right)\right|=\left|\uparrow a_{i}\right|^{2}+\left|\perp_{a} a_{i}\right|^{2}+1 \leqslant n^{2}-6 n+14
$$

For $n \geqslant 4$ the inequality $n^{2}-6 n+14 \leqslant(n-2)^{2}+2$ holds, and we obtain $(20)$.

Using $(3,4,19,20)$, we obtain

$$
\begin{aligned}
\sum_{s \in S}\left|\operatorname{cov}_{1}(s)\right|=\mid & \operatorname{cov}_{1}(0)|+| \operatorname{cov}_{1}(1)|+| \operatorname{cov}_{1}(a)\left|+\sum_{s \notin\{0, a, 1\}}\right| \operatorname{cov}_{1}(s) \mid \leqslant n^{2}+n+(n-1)^{2}+1+ \\
& +(n-3)\left((n-2)^{2}+2\right)=n^{2}+n+(n-2)\left((n-2)^{2}+4\right)=\sum_{s \in F_{n}}\left|\operatorname{cov}_{1}(s)\right|
\end{aligned}
$$

that concludes the theorem.

\section{Discussing the conjecture}

In Introduction we mentioned the following conjecture.

Conjecture. Let $\Sigma(S)$ has the minimum value $\Sigma$ on the set $\mathbf{S}_{n}$. Then there exists a semilattice $S_{1} \in \mathbf{S}_{n}$ with a unique co-atom and $\Sigma\left(S_{1}\right)=\Sigma$.

Surprisingly, the linearly ordered semilattice $L_{n}$ has not the minimal value $\Sigma(S)$ in $\mathbf{S}_{n}$. For example, $\Sigma(S)$ has the minimum at the following semilattice $S_{5}$ in $\mathbf{S}_{5}$ (Fig. 2).

The number at each vertex $s$ is $\left|\operatorname{cov}_{1}(s)\right|$, but the linearly ordered semilattice $L_{5}$ has the following values of $\left|\operatorname{cov}_{1}(s)\right|$ (Fig. 3).

We have

$$
\Sigma\left(L_{5}\right)=25+17+11+7+5>25+13+13+7+5=\Sigma\left(S_{5}\right) .
$$

Using a computer, we checked the conjecture for each $n \in[4,10]$.

The author was supported by Russian Science Foundation (project 17-11-01117). 


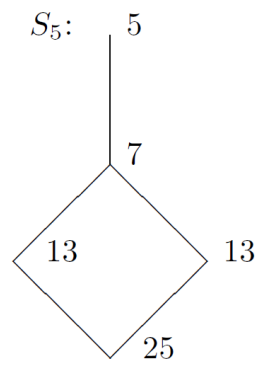

Fig. 2. The semilattice $S_{5}$

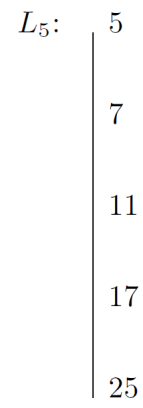

Fig. 3. The semilattice $L_{5}$

\section{References}

[1] A.Shevlyakov, Equivalent equations in semilattices, Sib. Electron. Mat. Izv., 13 (2016), 478-490 (in Russian).

\section{Эквационально экстремальные полурешетки}

Артем Н. Шевляков Омский филиал Института математики им. С.Л. Соболева СО РАН

Певцова, 13, Омск, 644099

Россия

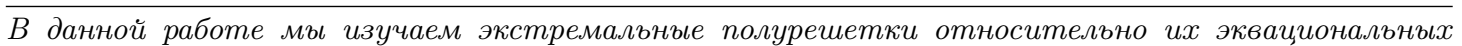
свойств. В классе $\mathbf{S}_{n}$ всех полурешеток порядка $n$ мы находим полурешетки с минималъным (максимальным) числом совместных уравнений. Кроме того, в $\mathbf{S}_{n}$ мы находим полурешетку с максимальной суммой числа решений всех уравнений.

Ключевые слова: полурешетки, уравнения, решения, совместность, универсалъная алгебраическая геометрия. 